\title{
Cambio del uso del suelo en el centro sur de Chile a fines del siglo XX. Entendiendo la dinámica espacial y temporal del paisaje
}

\author{
Land use change in the south central Chile at the end of the $20^{\text {th }}$ century. \\ Understanding the spatio-temporal dynamics of the landscape
}

\author{
MAURICIO AGUAYO ${ }^{1,3, *}$, ANÍBAL PAUCHARD ${ }^{2,3,4}$, GERARDO AZÓCAR ${ }^{1,3}$ \& OSCAR PARRA ${ }^{1,3}$ \\ ${ }^{1}$ Centro de Ciencias Ambientales EULA-Chile, Universidad de Concepción, casilla 160-C, Concepción, Chile \\ 2 Facultad de Ciencias Forestales, Universidad de Concepción, casilla 160-C, Concepción, Chile \\ ${ }^{3}$ Centro de Investigación en Ecosistemas de la Patagonia (CIEP) \\ ${ }^{4}$ Instituto de Ecología y Biodiversidad (IEB) \\ * Autor correspondiente: maaguayo@udec.cl
}

\begin{abstract}
RESUMEN
Los humanos han transformado gran parte de la superficie del planeta en los últimos 300 años. La tala de bosques, las prácticas agrícolas de subsistencia, la intensificación de la producción agrícola y la expansión de los centros urbanos han cambiado drásticamente el paisaje. En el centro y sur de Chile las transformaciones han estado asociadas, en un inicio, a la expansión de la frontera agropecuaria que permitió satisfacer tanto las necesidades internas como la creciente demanda externa por productos agrícolas. Posteriormente, un fuerte incentivo a la forestación, comandado por el Estado, generó un acelerado proceso de desarrollo forestal que explica, en gran medida, la actual configuración del paisaje en esta región. En este trabajo hemos analizados la dinámica espacial de los cambios en el uso del suelo ocurridos durante las últimas décadas en un área de 2.3 millones de hectáreas ubicada entre las regiones del Biobío y La Araucanía. Las coberturas del suelo fueron obtenidas a partir de dos imágenes Landsat correspondientes a los años 1979 y 2000 . Los resultados confirman que las principales transformaciones del paisaje son consecuencia del desarrollo forestal, la mantención de los rubros agropecuarios y el crecimiento urbano e industrial. Asimismo, fue posible determinar que la pérdida del bosque nativo se debe, principalmente, a la expansión de las plantaciones forestales hacia los cordones montañosos andinos y costeros.
\end{abstract}

Palabras clave: cambio de uso del suelo, Chile, desforestación, dinámica del paisaje.

\begin{abstract}
Land use changes have transformed much of the Earth's surface in the last 300 years. Deforestation, intensification of agriculture, and urban sprawl have radically changed the preindustrial landscape. In southcentral Chile, landscape change was initially related to the expansion of farming to address national needs and the growing international demand for agricultural products. Subsequently, private forestry, subsidized by the State, promoted the accelerated expansion of industrial plantations, a process that largely determines the current landscape configuration. In this study, we document the spatial dynamics of land-use change in 2.3 million hectares of the Biobío and Araucanian regions during the last two decades of the $20^{\text {th }}$ century. Two land cover maps were developed from satellite imagery comparing a 20-year period (1979 and 2000). Results confirm that major changes in the landscape were due to exotic tree plantations for forestry purposes, agricultural activities, and urban and industrial sprawl. Accordingly, we conclude that substantial loss of native forest cover was driven by the expansion of commercial plantations towards mid-elevations in the Andes and Coastal ranges.
\end{abstract}

Key words: dynamic landscape, land use and land cover change, native forest loss, south central Chile. 


\section{INTRODUCCIÓN}

La magnitud, extensión y velocidad de las alteraciones antropogénicas sobre la superficie de la tierra no tienen precedentes en la historia de la humanidad (Lambin et al. 1999, NRC 2001). Alrededor de la mitad de la superficie de la tierra ha sido directamente transformada por la acción humana (Vitousek et al. 1997). Estos cambios han sido tan dramáticos que se acercan a los niveles de transformación que ocurrieron durante los períodos glaciales (NRC 2001). Goldewijk (2001) ha determinado que durante el período 1700-1990, 1,206 millones de hectáreas fueron convertidas en terrenos agrícolas y 2,927 millones fueron transformadas en áreas de pastoreo. Por su parte, Ramankutty \& Foley (1999) estimaron que, entre 1700 y 1992, 1,621 millones de hectáreas fueron habilitadas para la agricultura de las cuales 885 millones correspondían a bosque, 565 millones a sabana/ praderas/estepa, 150 millones a matorrales y 21 millones a tundras/desiertos. Debido a estas transformaciones las tierras de cultivo y pastoreo son, en la actualidad, el bioma terrestre más grande del planeta el cual ocupa alrededor de 40 $\%$ de la superficie de la tierra (Ramankutty \& Foley 1999, Asner et al. 2004). Mundialmente, las actividades agropecuarias, junto con la extracción de madera, han causado una pérdida neta de 7 a 11 millones de $\mathrm{km}^{2}$ de bosque en los últimos 300 años (Ramankutty \& Foley 1999, Foley et al. 2005).

El cambio de uso del suelo se ha convertido en un importante forzante del cambio climático regional y global (Houghton et al. 1999, Chase et al. 2000, Claussen et al. 2001, DeFries et al. 2002, Brovkin et al. 2004); es considerado la primera causa de alteración del suelo (Matson et al. 1997, Lambin et al. 2001, Foley et al. 2005); y afecta, fuertemente, la capacidad de los sistemas biológicos para soportar y satisfacer las necesidades humanas (Vitousek et al. 1997, Foley et al. 2005). Tales cambios incrementan, a su vez, la vulnerabilidad de ecosistemas y personas frente a desastres naturales (Lambin et al. 2001). Sin embargo, la pérdida de biodiversidad es el impacto más evidente y directo generado por la transformación de las principales coberturas naturales. La pérdida, modificación y fragmentación de hábitats han provocado un fuerte declive de la biodiversidad a nivel mundial (Pimm \& Raven 2000, Sala et al 2000). Según Sala et al. (2000), el cambio de uso del suelo es el factor que se espera tenga el impacto global más importante sobre la biodiversidad al año 2100, principalmente, debido a sus devastadores efectos sobre la disponibilidad de hábitat y extinción de especie. Se estima que durante el último siglo, la mayor parte de los ecosistemas mundiales fueron afectados por el cambio de uso de la tierra (Vitousek et al. 1997). La mayoría de los impactos se han producido por la pérdida y/o transformación de ecosistemas boscosos y praderas naturales en terrenos habilitados para el desarrollo agrícola, ganadero, forestal y urbano/industrial (Sala et al. 2000).

En Chile, los primeros estudios sobre las transformaciones antropogénicas del paisaje se realizaron en la zona central a fines de la década de los setenta (Fuentes \& Hajek 1979). Durante la década de los ochenta, nuevas contribuciones aportaron al conocimiento de los cambios en el uso del suelo ocurridos en el centro y sur del país (Fuentes \& Carrasco 1984, Fuentes1988, Lara et al. 1989). En la década de los noventa, el gobierno de Chile, a través de la Corporación Nacional Forestal (CONAF) y la Comisión Nacional del Medio Ambiente (CONAMA), realiza un gran esfuerzo para cuantificar los recursos vegetacionales nativos existentes en el país e inicia una serie de monitoreos para detectar los cambios de cobertura (CONAF 1999a, 1999b). Recientemente, debido a la disponibilidad de imágenes satelitales, los estudios de cambio en la cobertura de suelo han ampliado la ventana temporal de análisis reconstituyendo paisajes de hace 30 años (e.g. Pauchard et al. 2006, Echeverría et al. 2006, Altamirano et al. 2007). Estas investigaciones dan cuenta de las transformaciones del paisaje a escala local y regional, y exploran sus consecuencias ecológicas y sociales. Localmente, se ha analizado el patrón de crecimiento urbano y evaluado sus efectos sobre coberturas de bosque nativo y humedales (Henríquez et al. 2006, Pauchad et al. 2006, Aguayo et al. 2007, Azócar et al. 2007). A escala regional, los estudios se han orientado a determinar las causas de la pérdida y fragmentación del bosque nativo e indagar sobre sus efectos (Wilson et al. 2005, Echeverría et al. 2006, 2007, Altamirano et al. 2007). Estos 
antecedentes muestran que las principales causas de transformación del paisaje en el centro y sur del país fueron en un inicio la habilitación de terrenos para la agricultura, y, posteriormente, la expansión de las plantaciones forestales incentivadas por un fuerte subsidio estatal (Donoso \& Lara 1996, Cisterna et al. 1999, CONAF et al. 1999b, Sanhueza \& Azócar 2000).

La evaluación de los efectos del cambio de uso del suelo sobre los ecosistemas terrestres depende en gran medida del conocimiento de las prácticas pasadas y la proyección de escenarios futuros (NRC 2001). El estudio histórico de los cambios en el uso del suelo permite entender la actual configuración del paisaje e identificar los impactos ambientales y sociales asociados a estas transformaciones (Andersen et al. 1996, Pan et al. 1999). El presente trabajo apunta a evaluar, en una amplia escala espacial y temporal, la dinámica de los cambios en el uso del suelo ocurridos en la zona centro sur de Chile. En particular, analizamos la dinámica espacial de las transformaciones del paisaje y cuantificamos los cambios de la cobertura del suelo en una de las regiones más pobladas e industrializadas del país, ubicada dentro de un área de alto valor para la biodiversidad a nivel mundial (Myers et al. 2000, Myers 2003), y en el límite de dos ecorregiones que presentan serios problemas de conservación (Olson \& Dinerstein 1998, Olson et al. 2001, Olson \& Dinerstein 2002).

\section{MÉTODOS}

\section{Área de estudio}

El área de estudio abarca una superficie de 2.4 millones de hectáreas y se encuentra ubicada entre las regiones del Biobío y La Araucanía en la zona centro sur de Chile $\left(36^{\circ} 33^{\prime} 06^{\prime}\right.$ " S $72^{\circ} 45^{\prime} 12^{\prime \prime}$ O y $38^{\circ} 14^{\prime} 23^{\prime \prime}$ S - 71³6’53” O; Fig. 1). En cuanto a sus características climáticas y ecológicas, el área se identifica como una zona de transición entre condiciones de dominio mediterráneo y templado húmedo. El efecto transicional del clima facilita el desarrollo de especies esclerófilas en su parte septentrional que dan paso a formaciones típicas del bosque templado del sur. Estas condiciones ambientales tienen directa relación con el aprovechamiento productivo del territorio que actualmente se encuentra dominado por una amplia superficie de plantaciones forestales basadas en especies exóticas de rápido crecimiento. El área es disectada por las cuencas de los ríos Itata y Biobío las que soportan un desarrollo industrial fuertemente ligado a la explotación forestal, producción agropecuaria y generación de energía. Además, se localiza en el límite de dos ecorregiones con prioridad de conservación a nivel mundial, el matorral mediterráneo y el bosque lluvioso templado (Olson \& Dinerstein 1998, Olson et al. 2001, Olson \& Dinerstein 2002, Myers et al. 2000, Myers 2003).

\section{Fuentes de información}

El análisis y cuantificación de los cambios de cobertura comprende una ventana temporal de 20 años. Los usos del suelo fueron derivados de la clasificación de dos imágenes Landsat cuyas escenas corresponden a los años 1979 (Landsat MSS) y 2000 (Landsat ETM+). Las escenas fueron obtenidas desde la galería de imágenes del Global Land Cover Facility (http:// www.landcover.org) cuyo nivel de procesamiento incluye correcciones radiométricas, geométricas y topográficas. Debido a que las imágenes presentan distintas resolución espacial (MSS: 79 m y ETM: 30 m), para comparar cuantitativamente los cambios en las coberturas de suelo, las imágenes fueron reagrupadas en celdas de $90 \times 90 \mathrm{~m}$.

\section{Coberturas del suelo}

Considerando que las imágenes no solo tienen diferencia en su resolución espacial sino también en la espectral se definieron nueve coberturas del suelo fácilmente identificables: (1) Bosque nativo (bosque adulto y renovales), (2) Matorral (matorral esclerófilo, matorral de especies exóticas, matorral nativo arborescente), (3) estepa andina, (4) plantaciones forestales (incluye áreas recientemente explotadas o plantadas), (5) terrenos agrícolas (incluye rotaciones cultivo/pradera), (6) cuerpos de agua (ríos, lagos y lagunas), (7) nieve, (8) áreas sin vegetación, y (9) áreas urbanas (incluye áreas industriales). Estas categorías corresponden a una simplificación y agregación del "Sistema General de Clasificación de la Vegetación" 
desarrollado para el proyecto "Catastro y Evaluación de los Recursos Vegetacionales Nativos de Chile" (CONAF et al. 1999a).

\section{Clasificación de imágenes satelitales}

El método de clasificación supervisada fue empleado en el procesamiento de las imágenes satelitales. Este método consistió en la selección de áreas representativas de cada cobertura del suelo para obtener su valor espectral. El criterio estadístico de máxima verosimilitud fue elegido para construir la firma espectral que permitió clasificar las imágenes según las categorías previamente definidas (Chuvieco 1996). Tres fuentes de información fueron utilizadas para seleccionar las áreas con las cuales se clasificó la imagen Landsat ETM: a) base de datos cartográfica del Catastro y Evaluación de los Recursos Vegetacionales Nativos de Chile (CONAF et al. 1999a), b) fotografías áreas digitales escala 1:115,000 de 1998 (CONAF et al. 1999b), y c) imágenes de alta resolución disponibles en Google Earth (http://earth.google.com). Estas fueron complementadas con 54 puntos de control en terreno para aquellas coberturas que presentaban mayor confusión. Las áreas utilizadas para clasificar la imagen Landsat MSS fueron seleccionadas a partir de zonas que, a través de una exhaustiva comparación visual de ambas imágenes (MSS y ETM), no

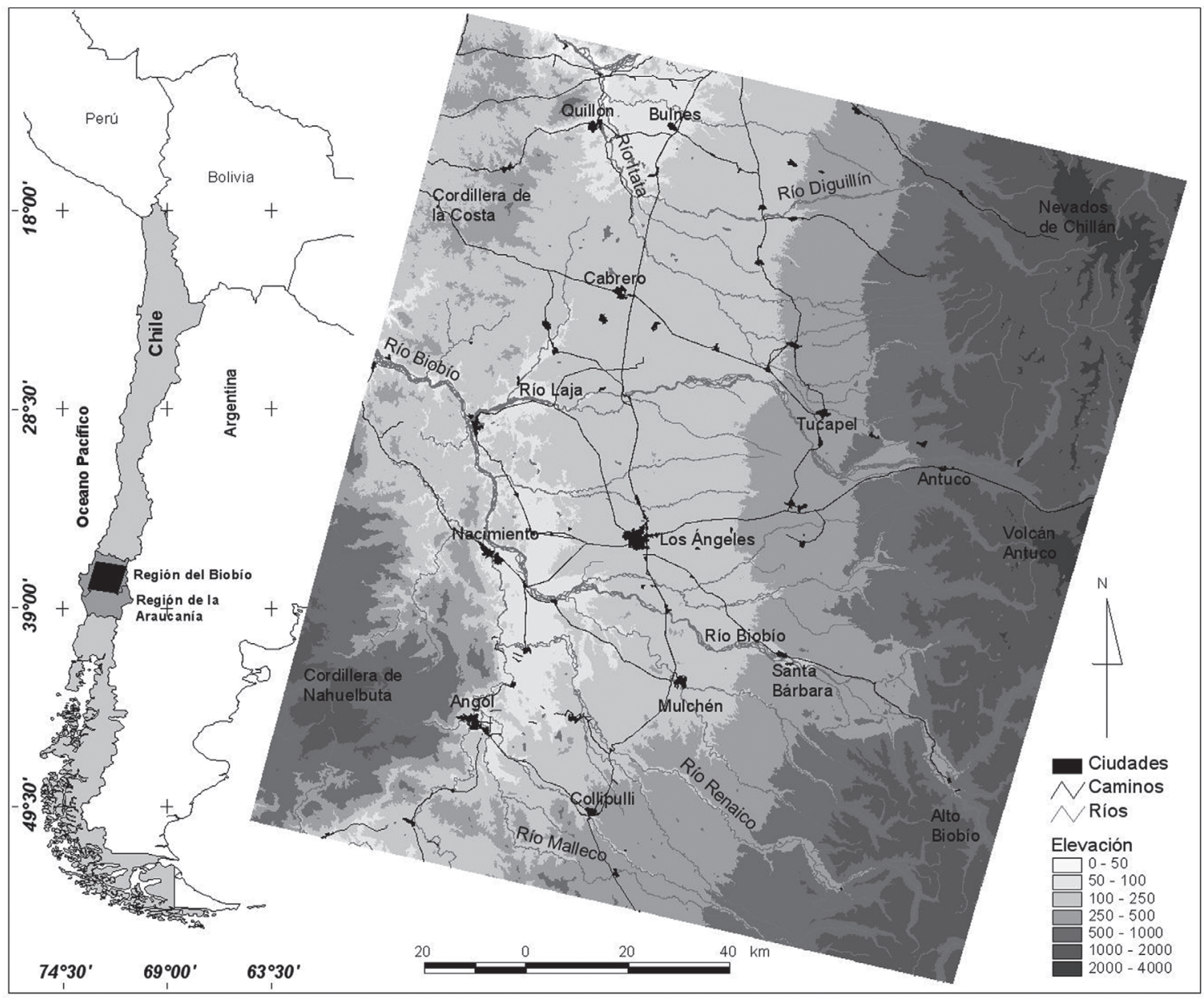

Fig. 1: Área de estudio. A la izquierda se muestra la localización del área analizada en un recuadro negro. A la derecha se muestran los principales centros poblados, caminos, ríos y rangos de elevación para facilitar la interpretación de los datos.

Study area. On the left side, the location of the area surveyed is shown inside a black box. On the right side, we see the cities, rivers, roads and elevation ranges for easier data interpretation. 
presentaron cambios. Esto fue complementado con fotografías áreas del Servicio Aerofotogramétrico de Chile (SAF) del año 1978 escala 1:60,000. Los software utilizados para la clasificación de las imágenes y análisis de los cambios fueron IDRISI 14.02 (Clark University, Worcester, Massachusetts, USA) y ArcGIS 9.0 (ESRI, Redlands, California, USA).

\section{Análisis y cuantificación de los cambios}

El cambio de uso del suelo fue cuantificado usando una matriz de transición que consiste en una tabla simétrica de doble entrada en cuyos ejes se presentan las coberturas del suelo y en cada celda la superficie de las áreas que experimentaron cambios entre los años 1979 y 2000. Las celdas situadas en la diagonal de la matriz contienen la superficie de aquellas áreas que no experimentaron cambios durante el período (Lopez et al. 2001, Aguayo et al. 2007). La matriz de transición fue construida a partir de la superposición y algebra de mapas.
Las tasas de cambio fueron calculadas utilizando la siguiente fórmula:

$$
\mathrm{P}=\frac{100}{\mathrm{t}_{2}-\mathrm{t}_{1}} \ln \frac{\mathrm{S}_{2}}{\mathrm{~S}_{1}}
$$

donde $S_{1}$ y $S_{2}$ corresponde a la superficie en el tiempo $\mathrm{t}_{1} \mathrm{y} \mathrm{t}_{2}$, respectivamente, y $\mathrm{P}$ es el porcentaje de cambio por año (FAO 1996, Echeverría et al. 2006).

\section{RESULTADOS}

El análisis de los datos de la matriz de transición reveló que alrededor del $40 \%$ del área de estudio ha sufrido modificaciones en casi todas las coberturas de suelo identificadas (Tabla 1). Las áreas que han permanecido sin alteraciones se concentraron, principalmente, a lo largo de la cordillera andina, en el sector norte del valle central y en la parte suroeste de la cordillera de la costa (Fig. 2). Las coberturas que

Matriz de transición. La suma de las columnas y filas corresponde al área total (en hectáreas) de cada cobertura en el año 1979 y 2000, respectivamente. La lectura de los valores en el sentido de las columnas indica el cambio de cobertura de suelo ocurrido entre el año 1979 y 2000; así por ejemplo, 132,388 ${ }^{\text {(a) }}$ hectáreas de bosque nativo, de un total de $654,069^{(\mathrm{b})}$ existentes el año 1979 , fueron convertidas en plantaciones forestales. Los valores de la diagonal corresponde a la superficie que no experimentó cambios durante el período; por ejemplo, $466,065^{(\mathrm{c})}$ hectáreas de terrenos agrícolas, de un total de $835,447^{\text {(d) }}$, se mantuvieron como tal entre 1979 y 2000.

Transition matrix. The total of each column and row indicates the area (in hectares) of land cover in the years 1979 and 2000, respectively. The values in the columns indicate land cover transitions occurred between 1979 and 2000 (e.g. $132,388^{(a)}$ hectares of native forest for a total of $654,069^{\text {(b) }}$ were converted to forest plantations). The values in the diagonal are the area that remained in the same categories during the study period (e.g. $466,065^{\text {(c) }}$ hectares of farmlands for a total of $835,447^{(\mathrm{d})}$ were maintained during the period 1979-2000).

\begin{tabular}{|c|c|c|c|c|c|c|c|c|c|c|}
\hline & \multicolumn{9}{|c|}{ Año 1979} & \multirow[b]{2}{*}{$\begin{array}{l}\text { Total } \\
2000\end{array}$} \\
\hline & $\begin{array}{l}\text { Bosque } \\
\text { Nativo }\end{array}$ & Matorral & Estepa & $\begin{array}{c}\text { Plantaciones } \\
\text { Forestales }\end{array}$ & $\begin{array}{l}\text { Terrenos } \\
\text { Agrícolas }\end{array}$ & $\begin{array}{l}\text { Cuerpos } \\
\text { de Agua }\end{array}$ & Nieve & $\begin{array}{c}\text { Áreas sin } \\
\text { Vegetación }\end{array}$ & $\begin{array}{l}\text { Áreas } \\
\text { Urbanas }\end{array}$ & \\
\hline Bosque nativo & 469380 & 0 & 0 & 0 & 0 & 0 & 0 & 0 & 0 & 469380 \\
\hline Matorral & 40138 & 162712 & 0 & 285 & 109135 & 416 & 0 & 1732 & 6 & 314425 \\
\hline Estepa & 0 & 0 & 130843 & 0 & 0 & 0 & 0 & 0 & 0 & 130843 \\
\hline Plantaciones forestales & $132388^{(a)}$ & 240421 & 0 & 78225 & 251295 & 415 & 0 & 2758 & 1 & 705503 \\
\hline$\overbrace{\text { }}^{8}$ Terrenos agrícolas & 10110 & 154487 & 0 & 23 & $466065^{(\mathrm{c})}$ & 241 & 0 & 585 & 15 & 631526 \\
\hline Cuerpos de Agua & 612 & 1798 & 0 & 2 & 897 & 5375 & 2 & 1978 & 0 & 10664 \\
\hline$\varangle_{\text {Nieve }}$ & 43 & 175 & 0 & 0 & 0 & 11 & 3602 & 2741 & 0 & 6572 \\
\hline Áreas sin Vegetación & 1221 & 6618 & 3 & 18 & 5028 & 1494 & 2053 & 49979 & 8 & 66422 \\
\hline Áreas urbanas & 178 & 1932 & 0 & 28 & 3026 & 3 & 0 & 62 & 4268 & 9497 \\
\hline Total 1979 & $654069^{(\mathrm{b})}$ & 568143 & 130847 & 78581 & $835447^{(d)}$ & 7955 & 5658 & 59836 & 4298 & 2344833 \\
\hline
\end{tabular}


experimentaron los mayores cambios corresponden a plantaciones forestales, terrenos agrícolas, matorrales y bosques nativos. En efecto, las plantaciones forestales aumentaron ocho veces la superficie registrada en el año 1979 (Tabla 2). En el caso de la cobertura agrícola, aun cuando el cambio neto fue negativo, la mayor parte de los suelos incorporados a esta actividad se atribuyen a la habilitación de terrenos cubiertos por matorrales. En dirección contraria, el abandono de terrenos agrícolas dio paso a una gran proporción de nuevas áreas cubiertas por matorrales (Tablas 1 y 2). Por su parte, los matorrales cedieron espacio a las plantaciones forestales y actividad agrícola disminuyendo su superficie casi a la mitad. En tanto la cobertura de bosque nativo registró una pérdida de más de un tercio de su superficie en 20 años (Tabla 2; Fig. 2).

\section{Plantaciones forestales}

El avance de las plantaciones forestales, que registraron el valor de ganancia más alto para el período (Tabla 2), explica gran parte de los cambios en las últimas décadas. El $40.1 \%$ del incremento neto ocurrió sobre terrenos agrícolas, el $38.3 \%$ ocupando áreas cubiertas
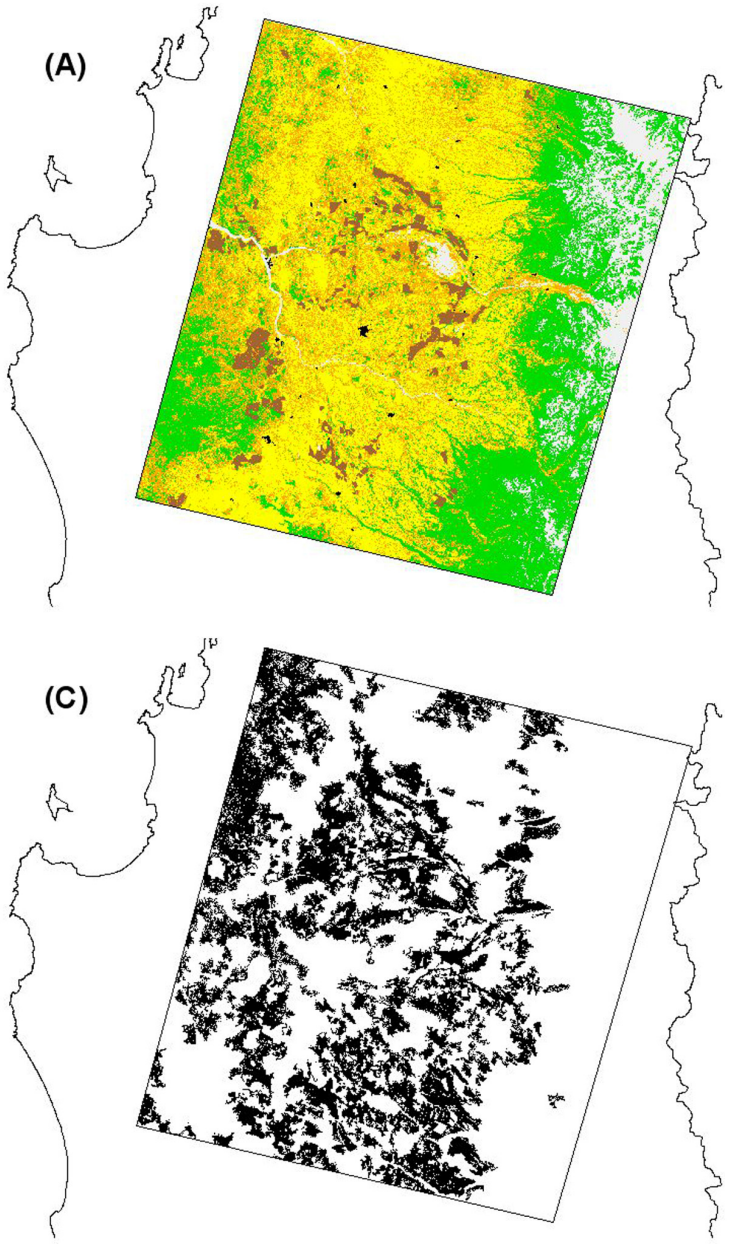

(B)

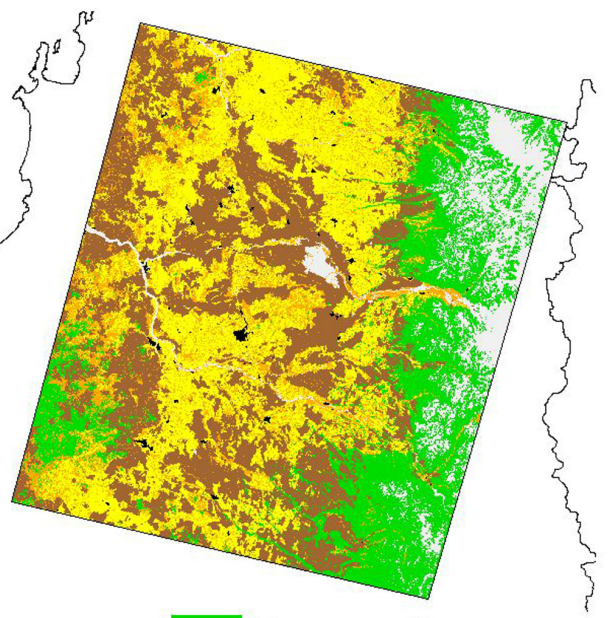

Bosque nativo Matorrales Plantaciones forestales Terrenos agrícolas Áreas urbanas Otras coberturas

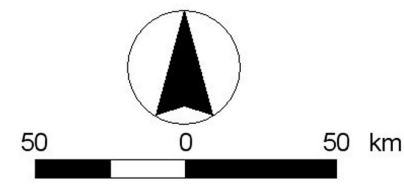

Fig. 2: Dinámica del uso de suelo: (A) coberturas de suelo año 1979, (B) coberturas de suelo año 2000 y (C) detección de cambios. La mayoría de los cambios ocurrieron en la ladera oriental de la cordillera de la costa, en el valle central y en la precordillera andina. Solo se muestran las áreas de cambios superiores a 500 hectáreas.

Land use dynamics: (A) land cover in 1979, (B) land cover in 2000 and (C) land cover changes. Most of the land cover changes occurred in the coastal range, central valley and Andes range. Only land cover changes greater than 500 hectares are shown. 
por matorrales y el $21.1 \%$ reemplazando el bosque nativo (Tabla 1; Fig. 3). En el año 1979 las plantaciones se distribuían, principalmente, en el valle central y en las laderas orientales de la cordillera de la costa. Hacia el año 2000 esta cobertura se extiende a través de todo el paisaje ocupando el $30 \%$ del área de estudio. De esta manera, las plantaciones forestales aumentaron a una tasa anual de $10.5 \%$ y son hoy una de las coberturas dominantes del paisaje (Tabla 2; Fig. 4).

\section{Terrenos agrícolas}

Durante el período de análisis esta cobertura registró una ganancia del $19.8 \%$ y una pérdida del $44.2 \%$ respecto a la superficie ocupada el año 1979 (Tabla 2). Esta dinámica generó una tasa de cambio negativa del $1.3 \%$ anual con una pérdida neta de $24.4 \%$ (Tabla 2). La pérdida de la cobertura agrícola fue producto principalmente de la forestación de estos terrenos. El $30 \%$ de los terrenos agrícolas existentes el año 1979 fueron incorporados a la actividad forestal durante el transcurso de los siguientes 20 años. En otras palabras, el $68 \%$ de la pérdida neta ocurrió a raíz de la forestación y el $29.5 \%$ debido al abandono de estos terrenos que, al año 2000, fueron ocupados por matorrales. El $2.5 \%$ restante han sido urbanizados o reemplazados por otras coberturas (Tabla 1; Fig. 3). Por otro lado, más del $90 \%$ de los terrenos agrícolas recuperados durante el período correspondieron a la habilitación de áreas cubiertas por matorrales (Tabla 1; Fig. 5).

\section{Matorrales}

Los matorrales registraron la mayor pérdida neta durante el período reduciendo su superficie en más del $40 \%$ respecto al año 1979 (Tabla 2). El $59 \%$ de la pérdida neta fue producto del reemplazo por plantaciones forestales y el $38 \%$ de la habilitación de terrenos agrícolas (Tabla 1; Fig. 3). En cuanto a la recuperación de esta cobertura, el $71.9 \%$ se debe al abandono de la actividad agrícola y el $26.5 \%$ a la transición del bosque nativo a matorrales (Tabla 1). Debido a estos procesos la dominancia del matorral en el paisaje disminuyó casi a la mitad aun cuando su distribución en pequeños fragmentos a través del paisaje no ha cambiado (Fig. 6).

\section{Bosque nativo}

Entre 1979 y 2000 fue posible cuantificar una pérdida neta de 184 mil hectáreas de bosque nativo que equivale al $28.2 \%$ del bosque existente en el año 1979. Durante este período el bosque nativo fue reemplazado a una tasa del $1.6 \%$ anual (Tabla 2). El $71.7 \%$ de las pérdidas fue producto de la sustitución por plantaciones forestales; el $21.7 \%$ pasó a la clase matorrales o renovales de baja altura; y el

TABLA 2

Pérdida, ganancia y tasa de cambio según coberturas de suelo entre 1979 y 2000.

Loss, gain and change rate by land cover types between 1979 and 2000 .

\begin{tabular}{lcccccccc}
\hline Coberturas de suelo & $1979(\mathrm{ha})$ & $(\%)$ & $2000(\mathrm{ha})$ & $(\%)$ & Tasa (\%) & $\begin{array}{c}\text { Pérdida } \\
\text { (ha) }\end{array}$ & $\begin{array}{c}\text { Ganancia } \\
\text { (ha) }\end{array}$ & $\begin{array}{c}\text { Cambio } \\
\text { neto (ha) }\end{array}$ \\
\hline Bosque Nativo & 654069 & 27.9 & 469380 & 20.0 & -1.6 & 184690 & 0 & -184690 \\
Matorral & 568143 & 24.2 & 314425 & 13.4 & -2.8 & 405431 & 151713 & -253718 \\
Estepa Andina & 130847 & 5.6 & 130843 & 5.6 & 0 & 3 & 0 & 3 \\
Plantaciones Forestales & 78581 & 3.4 & 705503 & 30.1 & 10.5 & 356 & 627278 & 626922 \\
Terrenos Agrícolas & 835447 & 35.6 & 631526 & 26.9 & -1.3 & 369382 & 165461 & -203921 \\
Cuerpos de Agua & 7955 & 0.3 & 10664 & 0.5 & 1.4 & 2580 & 5289 & 2709 \\
Nieve & 5658 & 0.2 & 6572 & 0.3 & 0.7 & 2056 & 2970 & 914 \\
Áreas sin Vegetación & 59836 & 2.6 & 66422 & 2.8 & 0.5 & 9856 & 16442 & 6586 \\
Áreas Urbanas & 4298 & 0.2 & 9497 & 0.4 & 3.8 & 30 & 5229 & 5199 \\
Total & 2344833 & 100.0 & 2344833 & 100.0 & & 974383 & 974383 & \\
\hline
\end{tabular}




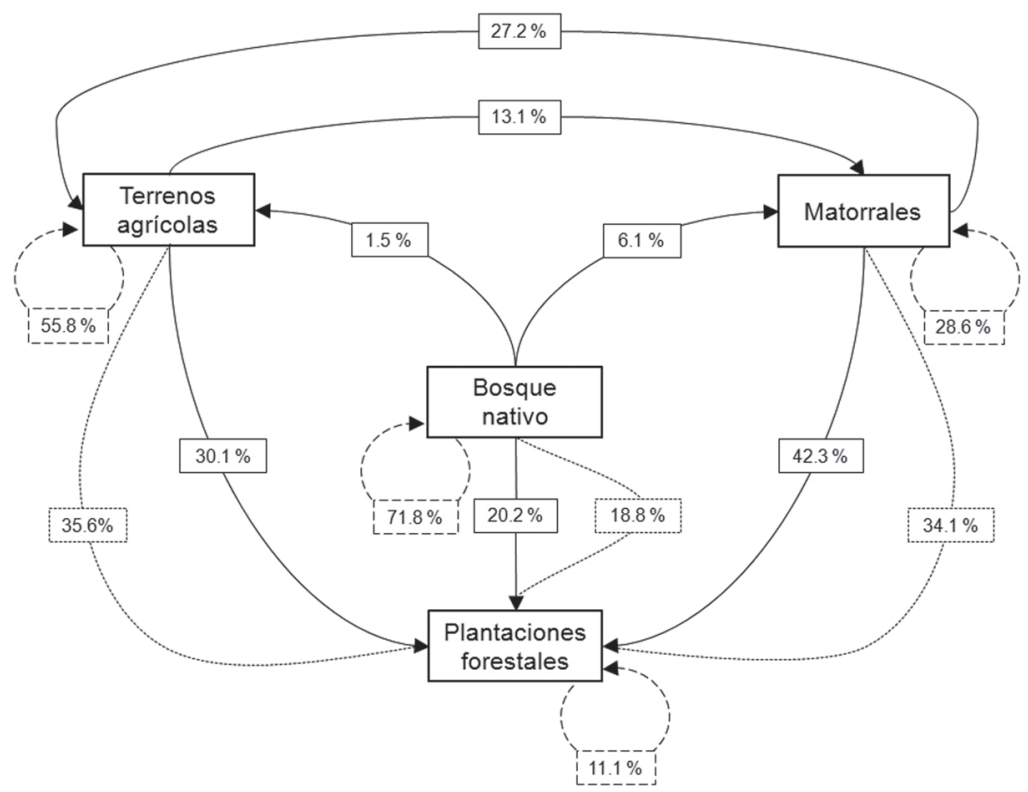

Fig. 3: Diagrama de transiciones entre coberturas de suelo durante el periodo 1979-2000. Línea continua indica el porcentaje de superficie perdida, línea segmentada indica el porcentaje de superficie sin cambios y línea de puntos indica el porcentaje de superficie ganada. Solo se incluyen porcentajes superiores al $1 \%$.

Flowchart transitions among land cover classes during the period 1979-2000. Continuous lines indicate losses (\%), segmented lines unchanged areas and dotted lines gains (\%). Only percentages $>1 \%$ are shown.
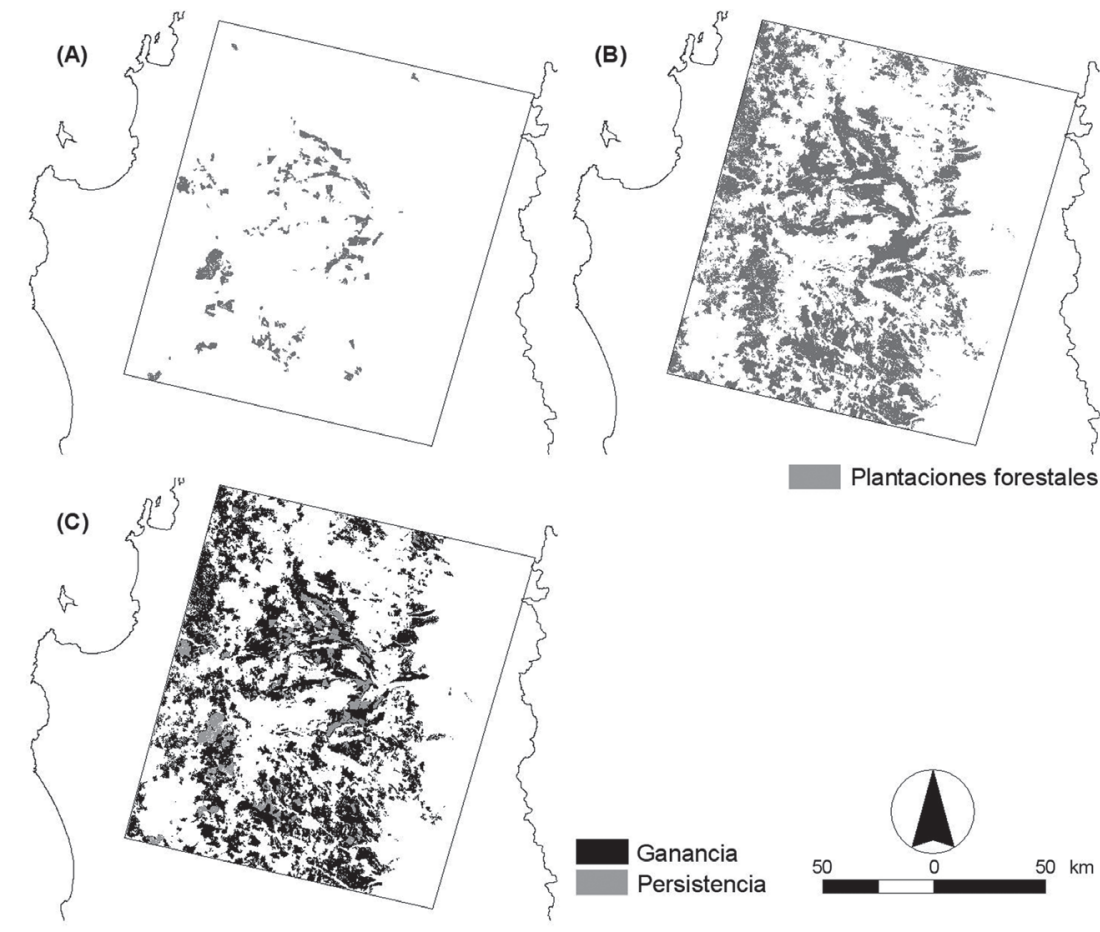

Plantaciones forestales

Fig. 4: Expansión de las plantaciones forestales durante el período 1979-2000: (A) plantaciones forestales en 1979, (B) plantaciones forestales en 2000 y (C) cambios detectados.

Expansion of forest plantations during the period 1979-2000: (A) forest plantations in 1979, (B) forest plantations in 2000 and (C) land cover change. 


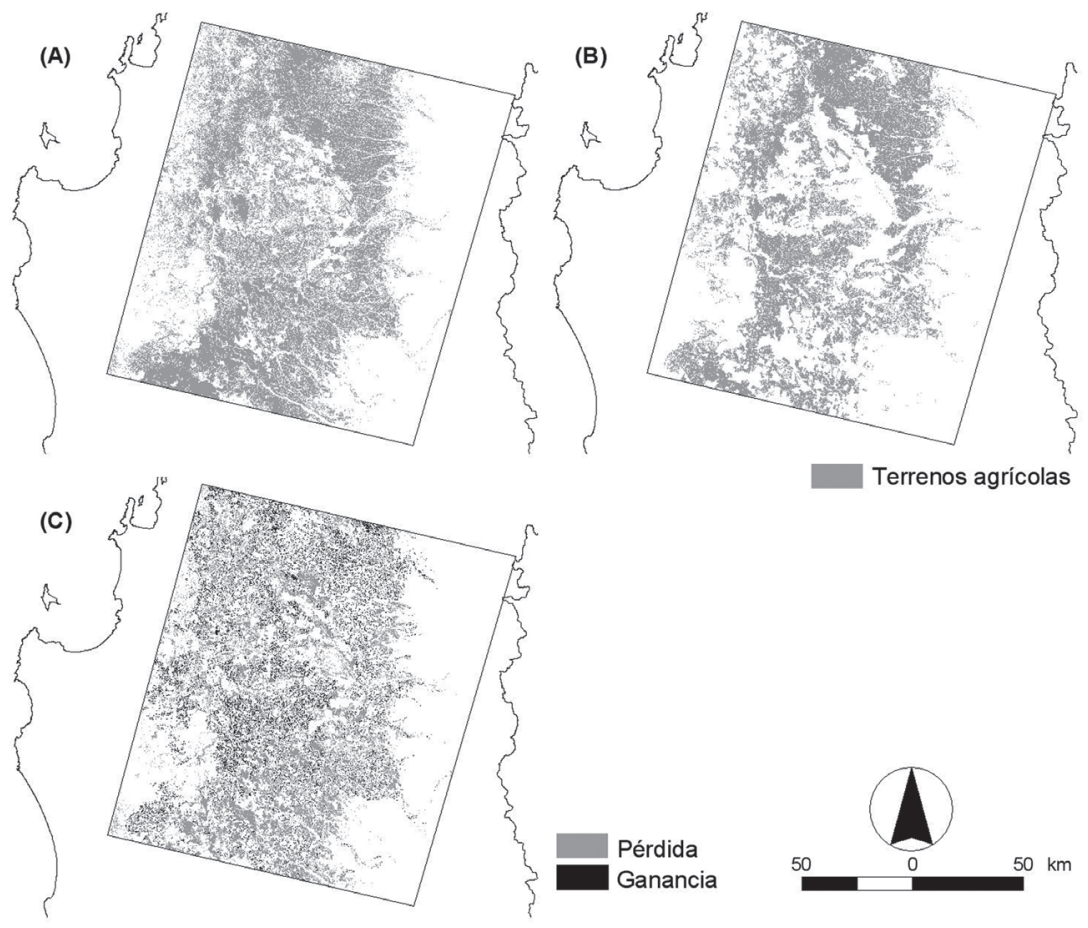

Fig. 5: Pérdida y ganancia de terrenos agrícolas durante 1979 y 2000: (A) terrenos agrícolas en 1979 , (B) terrenos agrícolas en 2000 y (C) cambios detectados.

Loss and gain of farmland during the period 1979-2000: (A) farmlands in 1979, (B) farmlands in 2000 and (C) land cover change.

$6.6 \%$ restante a terrenos agrícolas, áreas sin vegetación, cuerpos de agua (embalses) y áreas urbanas (Tabla 1; Fig. 3). Los cambios observados se distribuyen, principalmente, a lo largo de las laderas orientales y occidentales de las cordilleras de la costa y de los Andes, respectivamente. De esta manera, las inmediaciones de la cordillera de Nahuelbuta, las áreas preandinas entre los ríos Chillán y Vergara, y los contrafuertes cordilleranos de la costa entre los ríos Itata y Laja son los sectores en donde el bosque nativo ha experimentado los mayores cambios (Fig. 7).

\section{Áreas urbanas}

Las áreas urbanas aumentaron a más del doble respecto a la superficie del año 1979 ocupando, fundamentalmente, terrenos agrícolas (57.9\%) y matorrales (36.9 \%). El resto de la urbanización se produjo sobre bosque nativo (3.4\%), áreas sin vegetación y plantaciones forestales (Tabla 1 y 2 ). Los principales incrementos se deben a la expansión de algunas ciudades tales como Bulnes, Cabrero, Laja, Los Ángeles, Nacimiento, Mulchén, Angol, Collipulli, entre otras. Cabe destacar la aparición de nuevas áreas en sectores donde en 1979 no existían, y el explosivo aumento del área urbana de pequeños centros poblados (Fig. $8)$. Este hecho puede ser atribuible a la instalación de nuevas industrias asociadas a actividades agrícolas y forestales (Azócar et al. 2003, 2007, Aguayo et al. 2007).

\section{DISCUSIÓN}

\section{Aspectos metodológicos}

La libre disposición y el fácil acceso a imágenes satelitales (e.g., Landsat), cuyas primeras escenas datan de comienzos de la década de los setentas, están generando una creciente motivación por investigaciones relacionadas con el cambio de uso del suelo en 


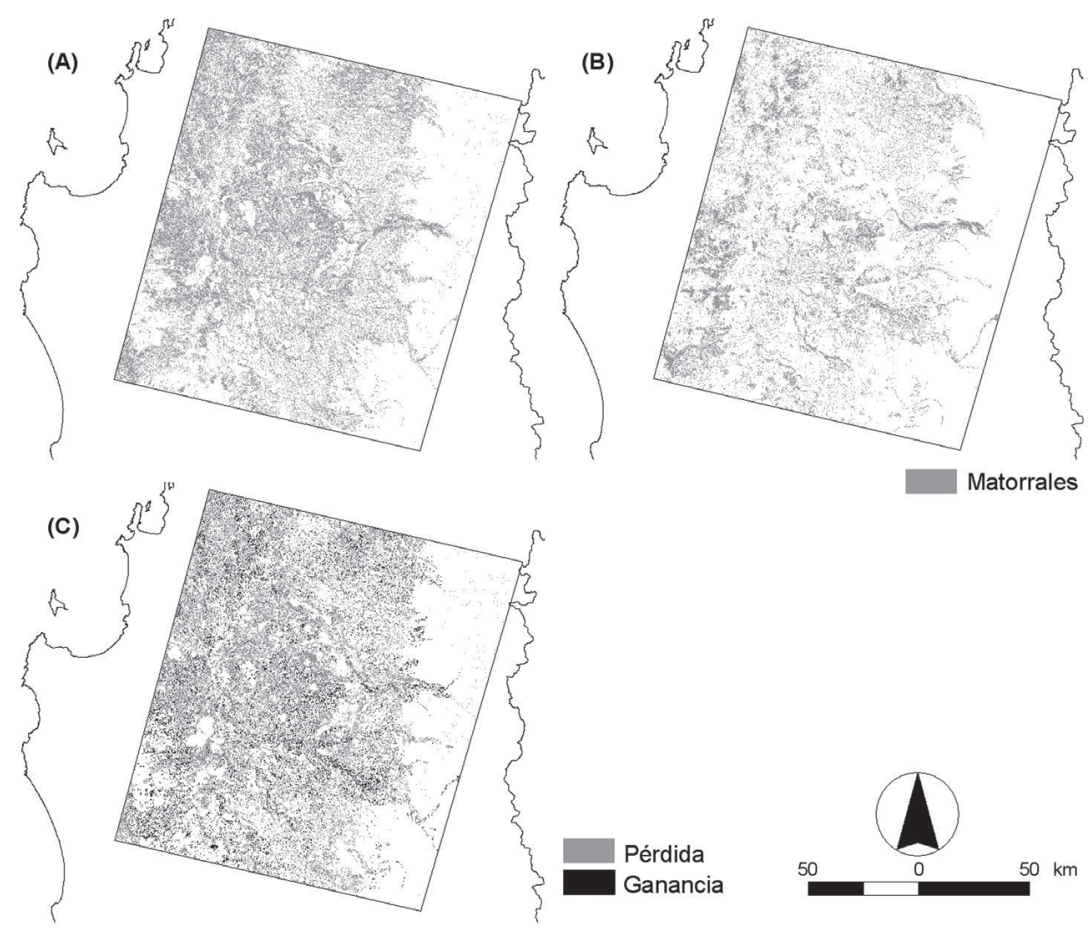

Fig. 6: Pérdida y ganancia de matorrales durante 1979 y 2000: (A) matorrales en 1979, (B) matorrales en 2000 y (C) cambios detectados.

Loss and gain of scrublands during the period 1979-2000: (A) scrublands in 1979, (B) scrublands in 2000 and (C) land cover change.

Chile. Los estudios se han orientado a detectar y cuantificar las trasformaciones del paisaje, describir su dinámica temporal y patrón espacial, e indagar en los efectos ecológicos a escalas local y regional (e.g. Pauchard et al. 2006, Echeverría et al. 2006, 2007, Altamirano et al. 2007). Esto da cuenta del gran potencial que tiene la utilización de imágenes satelitales multitemporales para el monitoreo de variables ambientales y ecológicas. En nuestro caso de estudio, las técnicas de análisis espacial y la disponibilidad de fuentes de información previa para calibrar y validar los resultados fueron apropiadas y suficientes para describir la dinámica del paisaje de un área representativa del centro sur de Chile. Es así como fue posible reconstituir la composición del paisaje para los años 1979 y 2000, cuantificar los cambios ocurridos durante el transcurso de 20 años e identificar la transición espacial de los mismos.

\section{Dinámica del paisaje}

Las principales fuerzas transformadoras del paisaje aquí analizado no difieren de las que otros autores ya han identificado en el centro sur de Chile (e.g. Donoso \& Lara 1996, Cisterna et al. 1999, Sanhueza \& Azócar 2000). De acuerdo a los resultados, los cambios más importantes se deben a la actividad agrícola y forestal. Distintos autores han señalado que la transformación inicial del paisaje fue producto de la habilitación de campos agrícolas a expensas de la tala o quema de la vegetación nativa que cubría gran parte del territorio (Donoso \& Lara 1996, Cisterna et al. 1999, Azócar \& Sanhueza 1999, Sanhueza \& Azócar 2000). En la cordillera de la costa extensas superficies de trigo reemplazaron al bosque nativo, y en el valle central y cordillera andina la vegetación natural fue aprovechada como combustible y talada para el establecimiento de cultivos y praderas (Millán \& Carrasco 1993).

A partir de la década de los treinta, en Chile comenzó el proceso de incentivo forestal apoyado por la promulgación de la Ley de Bosques de 1931 en cuyo cuerpo legal queda de manifiesto el interés del estado por promover la reforestación para el control de la erosión. Pero no fue hasta la década de los setenta cuando 


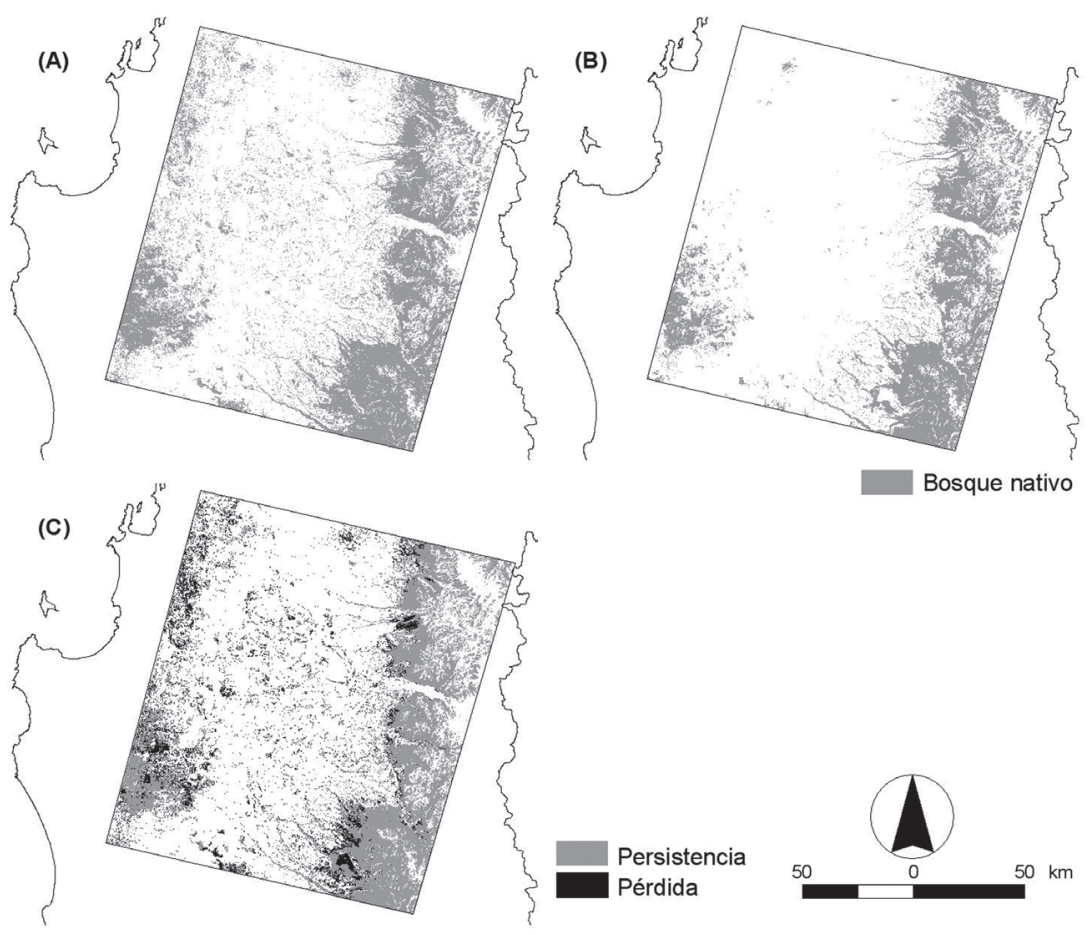

Fig. 7: Pérdida de bosque nativo durante el período 1979-2000: (A) bosque nativo en 1979, (B) bosque nativo en 2000 y (C) cambios detectados.

Native forest loss during the period 1979-2000: (A) native forest in 1979, (B) native forest in 2000 and (C) land cover change.

comienza la expansión progresiva de la actividad forestal basada en plantaciones de especies exóticas de rápido crecimiento (Cisterna et al. 1999, Pellet et al. 2005). En 1974 la promulgación del Decreto Ley 701, que subsidia la forestación y el manejo de nuevas plantaciones, da un decisivo impulso al desarrollo forestal (Millán \& Carrasco 1993, Donoso \& Lara 1996). El acelerado aumento de las plantaciones forestales que experimentó el país a partir de 1975 se debe, en gran medida, a la entrada en vigencia de esta Ley (Millán \& Carrasco 1993, Donoso \& Lara 1996, Camus \& Hajek 1998, Cisterna et al. 1999). La tasa de forestación anual se incrementó desde $48 \mathrm{mil}$ hectáreas en el período 1965-1974 a 83 mil entre 1975 y 1991 (Millán \& Carrasco 1993).

Entre 1979 y 2000 el $64 \%$ de los cambios ocurridos en el área de estudio fue producto del avance de las plantaciones forestales sobre terrenos agrícolas, matorrales y bosque nativo a una tasa anual del $10.5 \%$, cifra superior a las tasas de forestación calculada entre 1974 y 1994 para el país y la región del Biobío. Si se considera que en el año 1974 la superficie de plantaciones forestales en Chile alcanzaba las 480 mil hectáreas (Millán \& Carrasco 1993) y veinte años más tarde cubrían una superficie de 2.1 millones de hectáreas (CONAF et al. 1999a), la tasa de incremento de las plantaciones forestales puede ser estimada en $7.4 \%$ anual. De la misma manera, la tasa forestación para la región del Biobío fue calculada en $7.3 \%$ anual basándose en la superficie de plantaciones existentes en los años 1974 (219 mil hectáreas) y 1994 (939 mil hectáreas) según los datos de Millán y Carrasco (1992) y CONAF et al. (1999c), respectivamente.

Los datos revelan que en el área de estudio más de 130 mil hectáreas de bosque nativo fueron sustituidas por plantaciones forestales. Según Lara et al. (1996), extensas superficies de bosque habrían sido sustituidas por plantaciones en distintas regiones del país transformándose en una de las principales causas de destrucción de estos ecosistemas. Este análisis es coincidente con los resultados del programa de monitoreo de la vegetación nativa implementado por el proyecto CONAF- 

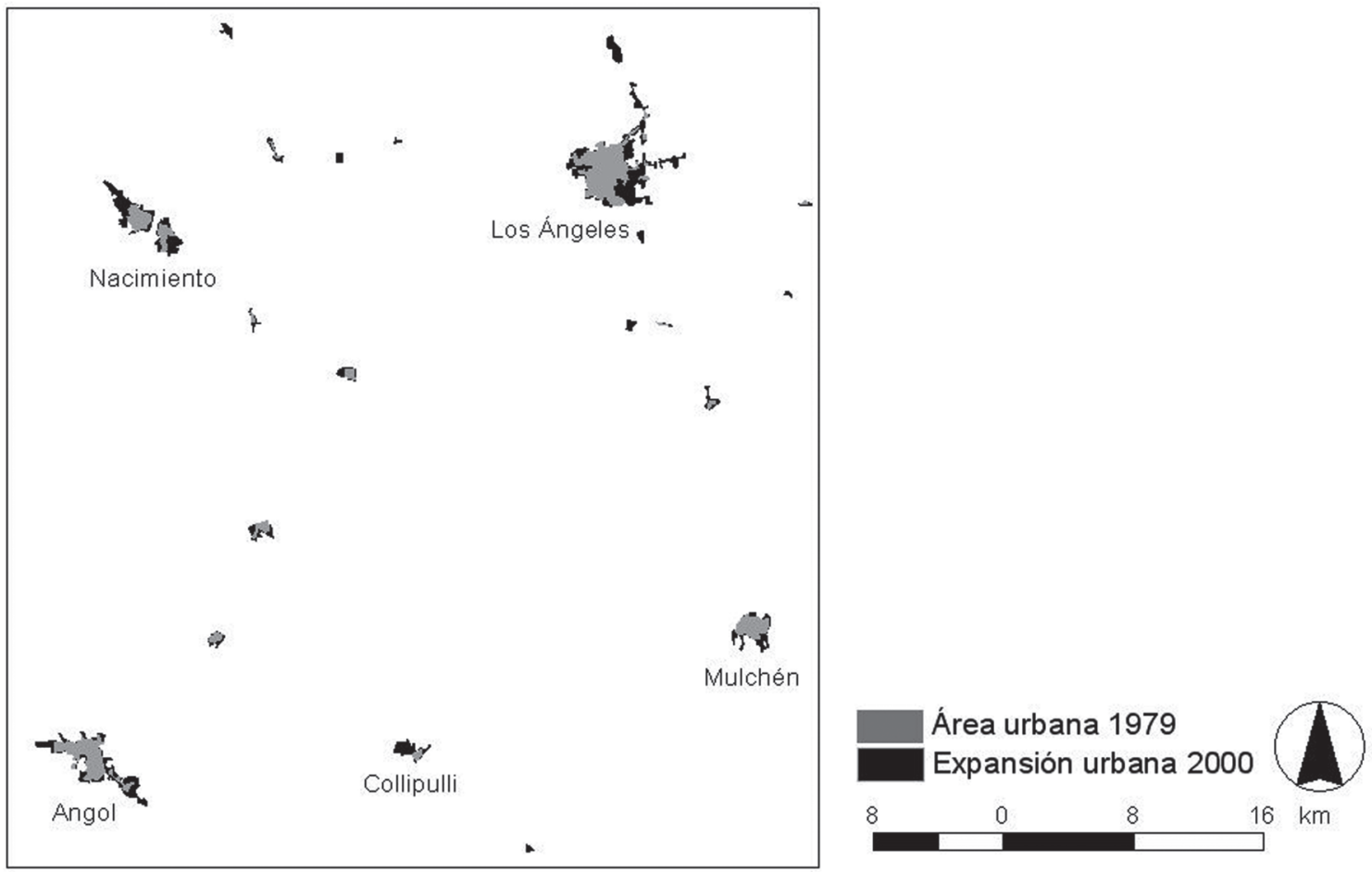

Fig. 8: Expansión urbana durante el período 1979-2000. Se muestra solo una sección del área de estudio debido a problemas de escala.

Urban sprawl during the period 1979-2000. Only one section of the study area is shown, because of scaling problems.

CONAMA-BIRF para la región del Biobío, el cual concluye que la disminución del bosque nativo, entre 1994 y 1998 , se debe mayoritariamente a la sustitución por plantaciones forestales (CONAF et al. 1999b). Se estima que entre 1974 y 1992 fueron sustituidas más de 200 mil hectáreas de bosque nativo en Chile (Lara et al. 1996). En las regiones del Maule y del Biobío 48 mil hectáreas de bosque nativo fueron reemplazadas por plantaciones de pino radiata durante el período 1978-1987 (Lara et al. 1989). En la Región de los Ríos, el 48.8 \% de las plantaciones efectuadas entre 1981 y 1993 (1,902 hectáreas) fueron establecidas sobre áreas anteriormente cubiertas por bosque nativo (Lara et al. 1996). Echeverría et al. (2006) reportaron para la cordillera de la costa, entre los ríos Maule y Cobquecura, la pérdida de 80 mil hectáreas de bosque nativo que durante los años 1975 y 2000 fueron reemplazadas por plantaciones forestales.

La tasa de pérdida de bosque nativo calculada para el área de estudio (1.6\% anual) difiere de las estimadas por CONAF et al. (1999b) y Echeverría et al. (2006). En efecto, Echeverría et al. (2006) calcularon una tasa de desforestación de $4.5 \%$ anual para el período 1975-2000 en un área de aproximadamente 500 mil hectáreas localizadas en la ladera occidental de la cordillera de la costa. Por su parte, la superficie de bosque nativo estimada por CONAF et al. (1999b) entre los años 1994 (786 mil hectáreas) y 1998 (777 mil hectáreas) permitió calcular una tasa de desforestación de $0.3 \%$ anual para la región de Biobío. Estas diferencias se deben, básicamente, a que la extensión y dinámica de los paisajes analizados son distintas. Las intervenciones de los cordones montañosos de los Andes y de la costa son más recientes que las ocurridas en el valle central (Millán \& Carrasco 1993, Cisterna et al. 1999, Azócar \& Sanhueza 1999). Este desfase en la evolución antrópica del paisaje explica las diferencias en las tasas de desforestación.

Aun cuando las tasas de desforestación del bosque nativo difieren de las obtenidas a partir de otras fuentes, los datos sugieren que la principal causa de pérdida del bosque nativo en 
la zona centro sur de Chile durante las últimas décadas es consecuencia de la expansión forestal. Las áreas de bosque nativo remanente se encuentran actualmente ubicadas en sectores de difícil acceso, fuertes pendiente, altitudes por sobre los $800 \mathrm{~m}$, o al interior de áreas silvestres protegidas.

Finalmente, durante el período de análisis se observó un aumento del área urbana superior al $50 \%$ respecto al año 1979 . La tasa de crecimiento anual calculada para el área de estudio $(3.8 \%)$ es similar a las estimadas para las ciudades de Chillán (3.1\%) y Los Ángeles (3.4\%) a partir de los trabajos de Azócar et al. (2003, 2007) y Henríquez et al. (2006). Al igual que lo ocurrido en otras ciudades intermedias de la zona central de país, el desarrollo urbano se produjo, especialmente, sobre áreas agrícolas (Azócar et al. 2003, 2007). La mayoría de las ciudades chilenas se han emplazado sobre áreas de alta productividad agropecuaria como lo confirman algunos autores (Soto \& Ulloa 1997, Henríquez et al. 2006, Aguayo et al. 2007, Azócar et al. 2007).

\section{AGRADECIMIENTOS}

Esta investigación ha sido financiada por el proyecto FONDECYT 1070511. A. Pauchard es parcialmente financiado por ICM 05-002 y PFB-23.

\section{LITERATURA CITADA}

AGUAYO M, T WIEGAND, G AZÓCAR, K WIEGAND \& C VEGA (2007) Revealing the driving forces of mid-cities urban growth patterns using spatial modeling: a case study of Los Ángeles, Chile. Ecology and Society 12: 13. (en línea) URL: http:// www.ecologyandsociety.org/vol12/iss 1/art 13/ (accedido Junio 20, 2009).

ALTAMIRANO A, C ECHEVERRÍA \& A LARA (2007) Efecto de la fragmentación forestal sobre la estructura vegetacional de las poblaciones amenazadas de Legrandia concinna (Myrtaceae) del centro-sur de Chile. Revista Chilena de Historia Natural 80: 27-42.

ANDERSEN O, TR CROW, SM LIETZ \& F STEARNS (1996) Transformation of a landscape in the upper mid-west, USA: The history of the lower St Croix River valley, 1830 to present. Landscape and Urban Planning 35: 247-267.

ASNER GP, AJ ELMORE, LP OLANDER, RE MARTIN \& AT HARRIS (2004) Grazing systems, ecosystem responses, and global change. Annual Review of Environment and Resources 29: 261-299.
AZÓCAR G \& R SANHUEZA (1999) Evolución del uso del suelo en las cuencas hidrográficas de las lagunas de la comuna de San Pedro de la Paz, región del Biobío: análisis histórico y tendencias. Revista Geográfica de Chile Terra Australis 44: 6378.

AZÓCAR G, R SANHUEZA \& C HENRÍQUEZ (2003) Análisis del cambio en los patrones de crecimiento urbano en una ciudad intermedia de Chile central: un caso de estudio en Chillán. Revista Latinoamericana de Estudios Urbano Regionales EURE 29: 79-82.

AZÓCAR G, H ROMERO, R SANHUEZA, C VEGA, M AGUAYO et al. (2007) Urbanization patterns and their impacts on social restructuring of urban space in Chilean mid-cities: The case of Los Ángeles, central Chile. Land Use Policy 24: 199-211.

BROVKIN V, S SITCH, W VON BLOH, M CLAUSSEN, E BAUER et al. (2004) Role of land cover changes for atmospheric $\mathrm{CO}_{2}$ increase and climate change during the last 150 years. Global Change Biology 10: $1253-1266$.

CAMUS P \& E HAJEK (1998) Historia ambiental de Chile. Descripción general de períodos en la historia ambiental y 15 estudios de casos de conflictos ambientales. Inscripción No. 106.166. I.S.B.N. 956-288-091-5. 183 pp.

CHASE TN, RA PIELKE, TGF KITTEL, RR NEMANI \& SW RUNNING (2000) Simulated impacts of historical land cover changes on global climate in northern winter. Climate Dynamics 16: 93-105.

CHUVIECO E (1996) Fundamentos de teledetección espacial. Tercera edición. Ediciones RIALP S.A., Madrid. 568 pp.

CISTERNA M, P MARTÍNEZ, C OYARZÚN \& P DEBELS (1999) Caracterización del proceso de reemplazo de vegetación nativa por plantaciones forestales en una cuenca lacustre de la cordillera de Nahuelbuta, VIII Región, Chile. Revista Chilena de Historia Natural 72: 541-556.

CLAUSSEN M, V BROVKIN \& A GANOPOLSKI (2001) Biogeophysical versus biogeochemical feedbacks of large-scale land cover change. Geophysical Research Letters 28: 1011-1014.

CONAF, CONAMA, BIRF, UNIVERSIDAD AUSTRAL DE CHILE, PONTIFICIA UNIVERSIDAD CATÓLICA DE CHILE et al. (1999a) Catastro y evaluación de los recursos vegetacionales nativos de Chile. Informe nacional con variables ambientales. Santiago, Chile. 88 pp.

CONAF, CONAMA, BIRF, UNIVERSIDAD AUSTRAL DE CHILE \& UNIVERSIDAD DE CONCEPCIÓN (1999b) Catastro y evaluación de los recursos vegetacionales nativos de Chile. Monitoreo de cambios. Santiago, Chile. 12 pp.

CONAF, CONAMA, BIRF, UNIVERSIDAD AUSTRAL DE CHILE, PONTIFICIA UNIVERSIDAD CATÓLICA DE CHILE et al. (1999c) Catastro y evaluación de los recursos vegetacionales nativos de Chile. Informe regional Octava Región. Santiago, Chile. 130 pp.

DEFRIES RS, L BOUNOUA \& GJ COLLATZ (2002) Human modification of the landscape and surface climate in the next fifty years. Global Change Biology 8: 438-458.

DONOSO C \& A LARA (1996) Utilización de los bosques nativos en Chile: pasado, presente y futuro. En: Armesto JJ, C Villagrán \& MK Arroyo (eds) Ecología de los bosques nativos de Chile: 363-387. Editorial Universitaria, Santiago, Chile. 
ECHEVERRÍA C, A NEWTON, A LARA, JM REYBENAYAS \& D COOMES (2007) Impacts of forest fragmentation on species composition and forest structure in the temperate landscape of southern Chile. Global Ecology and Biogeography 16: 426439.

ECHEVERRÍA C, D COOMES, J SALAS, JM REYBENAYAS, A LARA et a1. (2006) Rapid deforestation and fragmentation of Chilean temperate forests. Biological Conservation 130: 481-494.

FAO (1996) Forest resources assessment 1990. Survey of tropical forest cover and study change process. Rome $\mathrm{N}^{\circ} 26.152 \mathrm{pp}$

FOLEY JA, R DEFRIES, GP ASNER, GC BARFORD, G BONAN et al. (2005) Global consequences of land use. Science 309: 570-574.

FUENTES E (1988) Sinopsis de paisajes de Chile central. En: Fuentes E \& S Prenafeta (eds) Ecología del paisaje en Chile central. Estudios sobre sus espacios montañosos: 17-27. Ediciones Universidad Católica de Chile, Santiago, Chile.

FUENTES ER \& M CARRASCO (1984) Problems of resource management and land use in two mountain regions of Chile. En: Di Castri F, FWG Baker \& M Hadley (eds) Ecology in practice: 315-330. Tycooly international, Dublin, Irlanda.

FUENTES ER \& ER HAJEK (1979) Patterns or landscape modification in relation to agricultural practice in central Chile. Environmental Conservation 6: 265271.

GOLDEWIJK KK (2001) Estimating global land use change over the past 300 years: The HYDE Database. Global Biogeochemical Cycles 15: 417433.

HENRÍQUEZ C, G AZÓCAR \& H ROMERO (2006) Monitoring and modeling the urban growth of two mid-sized Chilean cities. Habitat International 30: 945-964.

HOUGHTON RA, JL HACKLER \& KT LAWRENCE (1999) The US carbon budget: Contributions from land-use change. Science 285: 574-578.

LAMBIN EF, $X$ BAULIES, $N$ BOCKSTAEL, $G$ FISCHER, T KRUG et al. (1999) Land-use and land-cover change (LUCC): Implementation strategy. IGBP Report No. 48, IHDP Report No. 10, Stockholm, Bonn.

LAMBIN EF, BL TURNER, HJ GEIST, SB AGBOLA, A ANGELSEN et al. (2001) The causes of land-use and land-cover change: moving beyond the myths. Global Environmental Change-Human and Policy Dimensions 11: 261-269.

LARA A, C DONOSO \& JC ARAVENA (1996) La conservación del bosque nativo en Chile: Problemas y desafíos. En: Armesto JJ, C Villagrán \& MK Arroyo (eds) Ecología de los bosques nativos de Chile: 335-362. Editorial Universitaria, Santiago, Chile.

LARA A, L ARAYA, J CAPELLA, M FIERRO \& A CAVIERES (1989) Evaluación de la destrucción y disponibilidad de los recursos forestales nativos en la VII y VIII Región. Informe Técnico, Comité Pro Defensa Fauna y Flora, Santiago, Chile. 22 pp.

LÓPEZ E, G BOCCO, M MENDOZA \& E DUHAU (2001) Predicting land-cover and land-use change in the urban fringe - A case in Morelia city, Mexico. Landscape and Urban Planning 55: 271-285.
MATSON PA, WJ PARTON, AG POWER \& MJ SWIFT (1997) Agricultural intensification and ecosystem properties. Science 277: 504-509.

MILLÁN J \& P CARRASCO (1993) La forestación en la VIII Región. Serie EULA Elementos cognoscitivos sobre el recurso suelo y consideraciones generales sobre el ordenamiento agroforestal. Editorial Universidad de Concepción, Concepción, Chile. $105 \mathrm{pp}$.

MYERS N (2003) Biodiversity hotspots revisited. BioScience 53: 916-917.

MYERS N, R MITTERNEIER, C MITTERNEIER, GB DA FONSECA \& J KENTS (2000) Biodiversity hotspots for conservation priorities. Nature 403: 853-858.

NRC (2001) Grand challenges in environmental sciences. Committee on Grand Challenges in Environmental Sciences. National Research Council, National Academy Press,Washington, DC, USA. 106 pp.

OLSON DM \& E DINERSTEIN (1998) The Global 200: A representation approach to conserving the Earth's most biologically valuable ecoregions. Conservation Biology 12: 502-515.

OLSON DM \& E DINERSTEIN (2002) The Global 200 Priority ecoregions for global conservation. Annals of the Missouri Botanical Garden 89: 199-224.

OLSON DM, E DINERSTEIN, ED WIKRAMANAYAKE, ND BURGESS, GVN POWELL et al. (2001) Terrestrial ecoregions of the world: A new map of life on Earth. BioScience 51: 933-938.

PAN DY, G DOMON, S DE BLOIS \& A BOUCHARD (1999) Temporal (1958-1993) and spatial patterns of land use changes in Haut-Saint-Laurent (Quebec Canada) and their relation to landscape physical attributes. Landscape Ecology 14: 35-52.

PAUCHARD A, M AGUAYO, E PEÑA \& R URRUTIA (2006) Multiple effects of urbanization on the biodiversity of developing countries: The case of a fast-growing metropolitan area (Concepción, Chile). Biological Conservation 127: 272-281.

PELLET P, E UGARTE, E OSORIO \& F HERRERA (2005) Conservación de la biodiversidad en Chile, ¿legalmente suficiente?: la necesidad de cartografiar la ley antes de decidir. Revista Chilena de Historia Natural 78: 125-141.

PIMM SL \& P RAVEN (2000) Biodiversity - Extinction by numbers. Nature 403: 843-845.

RAMANKUTTY N \& JA FOLEY (1999) Estimating historical changes in global land cover: Croplands from 1700 to 1992. Global Biogeochemical Cycles 13: $997-1027$

SALA OE, FS CHAPIN, JJ ARMESTO, E BERLOW, J BLOOMFIELD et al. (2000) Biodiversity - Global biodiversity scenarios for the year 2100 . Science 287: $1770-1774$.

SANHUEZA R \& G AZÓCAR (2000) Transformaciones ambientales provocadas por los cambios económicos de la segunda mitad del siglo XIX; provincia de Concepción. Revista Geográfica de Chile Terra Australis 45: 181-194.

VITOUSEK PM, HA MOONEY, J LUBCHENCO \& JM MELILLO (1997) Human domination of Earth's ecosystems. Science 277: 494-499.

WILSON K, A NEWTON, C ECHEVERRÍA, C WESTON \& M BURGMAN (2005) A vulnerability analysis of the temperate forests of south central Chile. Biological Conservation 122: 9-21. 